\title{
Native valve endocarditis and femoral embolism due to Granulicatella adiacens: a rare case report
}

Authors

Vandana KE ${ }^{1}$

Mukhopadhyay $\mathrm{C}^{2}$

Rau NR ${ }^{3}$

Ajith $\mathrm{V}^{4}$

Rajath $\mathrm{P}^{5}$

${ }^{1} \mathrm{MD}$ - Associate

Professor, Department of Microbiology, Kasturba Medical College, Manipal

University, Manipal, India.

${ }^{2} \mathrm{MD}$, PDCC - Professor, Department of

Microbiology, Kasturba

Medical College, Manipal

University, Manipal, India.

${ }^{3} \mathrm{MD}$ - Professor and Unit

Head, Department of

Medicine, Kasturba Medical

College, Manipal University,

Manipal, India.

${ }^{4}$ MBBS - Post Graduate

Student(MD), Department

of Microbiology, Kasturba

Medical College, Manipal

University, Manipal, India.

${ }^{5}$ Post Graduate

Student(MSc),

Department of

Microbiology, Kasturba

Medical College, Manipal

University, Manipal, India.

Submitted on: 03/16/2010 Approved on: 04/08/2010

\section{Correspondence to:} Dr.Vandana KE Associate Professor Dept of Microbiology Kasturba Medical College, Manipal, Manipal

University

Karnataka, India-576104

E-mail: vandanake@

gmail.com

We declare no conflict of interest.

\begin{abstract}
The fastidious Gram-positive cocci Granulicatella adiacens, previously known as nutritionally variant streptococci (NVS) are unusual but significant cause of endocarditis due to increased mortality and morbidity. Difficulties in reaching correct bacteriological identification, increased resistance to beta-lactam antibiotics and macrolides and complicated clinical course have contributed to problems in management of cases of infective endocarditis caused by this bacterium. We present the first Indian case of endocarditis with arterial embolus by $G$. adiacens in an elderly male with no preexisting cardiac abnormality.
\end{abstract}

Keywords: endocarditis; Granulicatella adiacens; femoral embolism.

[Braz J Infect Dis 2010;14(6):634-636]@Elsevier Editora Ltda.

\section{INTRODUCTION}

Infective endocarditis (IE) is a life-threatening disease of the endocardium. Despite advances in diagnosis, therapy and surgical techniques, high morbidity and mortality rates are observed related to this condition. ${ }^{1}$ The most predominant pathogens of IE are the bacterial species in the oral cavity such as the viridans group of streptococci. IE due to one of the most fastidious bacteria Granulicatella adiacens, formerly described as a member of nutritionally variant streptococci (NVS) creates problems in diagnosis and management due to difficulties in reaching early bacteriological confirmation and high prevalence of beta-lactam and macrolide resistance.,3 Granulicatella spp. are commonly found in the oral cavity, genitourinary and intestinal tract accounting for up to $6 \%$ of all streptococcal endocarditis along with Abiotrophia. ${ }^{4}$ We report the first Indian case of native valve endocarditis with femoral embolism in an elderly patient without any underlying cardiac abnormalities along with brief a review of prevalence, risk factors, management and outcome of IE due to Granulicatella spp.

\section{CASE REPORT}

A 71-year old male agricultural worker with no premorbid illness presented with intermittent low grade fever for 2.5 months and bilateral tingling and numbness in lower limbs for 15 days. He also had burning micturition for 5 days. He was in the habit of alcohol consumption and smoking but had stopped once the symptoms began. On examination, he was stable with BP - 130/80, PR - 82/min; heart sounds were heard with systolic murmur, normal respiratory and central nervous system. He did not have any gastrointestinal symptoms. No organomegaly was noted. Laboratory tests revealed hemoglobin $11.2 \mathrm{~g} / \mathrm{dL}$, leucocyte total count of 12,400 cells $/ \mathrm{mm}^{3}$ (polymorphs $87.9 \%$, lymphocytes $6.6 \%$, monocytes $3.2 \%$, eosinophils $2.2 \%$ ), platelets 221,000 cells $/ \mathrm{mm}^{3}$, ESR-95 mm/hr, normal urine microscopy, normal lipid profile except for lowered HDL cholesterol (17 mg/dL), normal liver and renal function tests and normal serum electrolytes. His chest $\mathrm{x}$-ray was normal. Echocardiography showed $2.0 \times 1.1 \mathrm{~cm}$ large mobile vegetation attached to the anterior mitral valve leaflet. Two sets of blood cultures were sent in a span of 24 hours using BacT/Alert system. All the 
samples flagged positive within 24 hours of incubation. Gram staining of positive blood cultures showed Grampositive cocci in chains. Cultures on 5\% sheep blood agar and chocolate agar incubated at $37^{\circ} \mathrm{C}$ in $\mathrm{CO}_{2}$ grew alpha hemolytic streptococci that was identified as Granulicatella adiacens by mini API Rapid ID32 Strept (bioMérieux, La Balme les Grottes, France). Antimicrobial susceptibility was performed using kirby Bauer disc diffusion method according to standard guidelines. ${ }^{5}$ The isolate was susceptible to ampicillin, erythromycin, cotrimoxazole, ciprofloxacin, gentamicin, cefotaxime and vancomycin. Diagnosis of infective endocarditis was made using modified Dukes criteria and the patient was started on ampicillin $12 \mathrm{~g} / 24 \mathrm{hrs}$ and gentamicin $180 \mathrm{mg} / 24 \mathrm{hrs}$. On the $6^{\text {th }}$ day of therapy he suddenly developed pain in the right lower limb raising suspicion of an embolic phenomenon. Arterial Doppler showed a $4.2 \mathrm{~cm}$ thrombus in the right common femoral artery causing complete occlusion of the common femoral artery, origin of the saphenophemoral artery and profunda femoris artery, that was subsequently embolectomised by Fogartys procedure. He was put on low molecular heparin for three days with continuation of antibiotic therapy for a total of five weeks. The patient was afebrile and symptomatically better during the rest of his hospital stay. Echocardiography repeated on day 27 showed complete clearance of vegetation. There was no relapse noticed on subsequent visits for follow-up.

\section{DISCUSSION}

Infective endocarditis remains a life-threatening disease. Continuous bacteremia in IE blood culture has made it the most important laboratory diagnostic test by recovery of etiologic agent. ${ }^{6}$ But sterile blood cultures in up to $31 \%$ of endocarditis has been noted due to various reasons like prior antibiotic therapy, right sided or mural endocarditis and endocarditis caused by slow growing or fastidious bacteria. Granulicatella spp along with Abiotrophia comprise up to $6 \%$ of all streptococcal endocarditis. ${ }^{4,6}$ Several reports of NVS endocarditis have appeared in literature, but the recent nomenclature of Granulicatella new genus in 2000 has made this new emerging fastidious bacteria of interest in terms of pathogenesis and management.

The Granulicatella genus has three species namely: G. adiacens, G. elegans and G. para-adiacens according to Collins and Lawson. ${ }^{7}$ Apart from being normal flora of oral cavity, genitourinary and intestinal tract, Granulicatella spp. cause infections such as endocarditis, bacteremia, keratoconjunctivitis, CNS infections, otitis, osteomyelitis, and pancreatic abscess. ${ }^{8}$ Rates of oral colonization by Granulicatella exceed that of Abiotrophia, prompting to assign oral cavity as the major source of infections. ${ }^{9}$ Fastidious nature of growth and high prevalence of beta-lactam and macrolide resistance may pose challenge to treat invasive infections. Most of the published reports suggest that infection by Granulicatella commonly occurred in patients with underlying cardiac disease or febrile neutropenia, while Abiotrophia infections occurred in immunocompetent individuals. Granulicatella bacteremia may occur in the setting of chemotherapy associated mucositis and neutropenia. Higher rates of orointestinal colonization by Granulicatella and subsequent mucositis may predispose for bacteremia in such patients. ${ }^{8}$

Preexisting cardiac pathology is a frequent risk factor in endocarditis due to Granulicatella. Published case reports or case series have shown that the elderly are more commonly affected than the younger age group. Prior dental manipulations in past six months have emerged as a contributory factor for IE in the majority of reports. Most commonly affected valves are aortic valves and mitral valves.., ${ }^{3,8-12}$ In most cases the disease course is slow and indolent. Embolizations occur in one third of patients in the absence of classical symptoms of digital clubbing, Osler's nodes and petechiae. In spite of in vitro susceptibility, treatment failure is observed in about $41 \%$ of cases, and nearly $27 \%$ require surgical intervention due to congestive cardiac failure and major systemic emboli. ${ }^{4}$ Life-threatening complications like recurrent infectious intracranial aneurysm with intracerebral hemorrhage as a sole presentation in silent IE in a young patient with no risk factors was also noted. This patient recovered completely only after mitral valvuloplasty. ${ }^{13}$ In another case report by Rosenthal et al., an elderly diabetic male patient had presented with endocarditis of a pacemaker lead along with vertebral osteomyelitis. The patient recovered with medical management alone..$^{14}$ All the cited cases of the literature describe endocarditis affecting native valves while only one report is available on prosthetic valve involvement by Granulicatella. ${ }^{15}$ High mortality is reported for endocarditis by NVS. Woo et al., in their case series of Granulicatella and Abiotrophia bacteremia, have reported 100\% mortality among those who had IE or infected atheroma by G. adiacens. ${ }^{12}$ Many reports have cited beta-lactams like ampicillin or ceftriaxone combined with gentamicin for 4-6 weeks in treating IE by Granulicatella spp leading to bacteriological cure. One report is available on treatment by ceftriaxone monotherapy failure leading to relapse was subsequently cured by combining of benzylpenicillin and gentamicin. ${ }^{10}$

Our case is in support of many published cases in terms of age, indolent clinical course and involvement of the mitral valve. Nonetheless, our patient did not have any underlying cardiac or immunosuppressive illness and did not undergo dental manipulations. The portal of entry of bacteria into the endovascular system remains obscure. But the observed poor dental hygiene might have facilitated the process towards IE. Though the etiological diagnosis was made within 48 hours of blood culture isolate and being sensitive to betalactams, the patient developed complications in the form of femoral thrombosis requiring surgical removal. However, complete cure was achieved with standard 4-week regimen of combination therapy of beta-lactam and gentamicin. 


\section{CONCLUSION}

Granulicatella is an important agent causing IE with increased morbidity and mortality, especially in elderly patients. Its fastidious nature of growth has led to difficulties in isolation and identification in clinical microbiology setups. Hence appropriate culture techniques and high suspicion index in differential identification of alpha-hemolytic streptococci are essential to reach appropriate diagnosis and plan management strategies.

\section{ACKNOWLEDGMENT}

We acknowledge the support of Dr. Indira Bairy, Professor and Head of the Department of Microbiology, in preparing the manuscript.

\section{REFERENCES}

1. Wilson W, Taubert KA, Gewitz M et al, Prevention of Infective Endocarditis Guidelines From the American Heart Association: A Guideline From the American Heart Association Rheumatic Fever, Endocarditis, and Kawasaki Disease Committee, Council on Cardiovascular Disease in the Young, and the Council on Clinical Cardiology, Council on Cardiovascular Surgery and Anesthesia, and the Quality of Care and Outcomes Research Interdisciplinary Working Group. Circulation. 2007; 116:1736-1754.

2. Tuohy MJ, Procop GW, Washington JA, Antimicrobial susceptibility of Abiotrophia adiacens and Abiotrophia defective. Diagn Microbiol Infect Dis. 2000; 38:189-191.

3. Liao CH, Teng LJ, Hsueh PR et al. Nutritionally variant streptococcal infections at a University Hospital in Taiwan: disease emergence and high prevalence of beta-lactam and macrolide resistance. Clin Infect Dis 2004; 38:452-455.

4. Brouqui P, Raoult D, Endocarditis due to rare and fastidious bacteria. Clin Microbiol Rev, 2001; 14:177-207.
5. Committee for Clinical Laboratory Standards Institute (Formerly National Committee for Clinical Laboratory Standards). 2005. Performance standards for antimicrobial disk susceptibility testing, fifteenth informational supplement. Approved standard M2-A8. National Committee for Clinical Laboratory Standards, Wayne, Pa.

6. Casalta JP, Habib G, La Scola B, Drancourt M, Caus T, Raoult D. Molecular diagnosis of Granulicatella elegans on the cardiac valve of a patient with culture-negative endocarditis. J Clin Microbiol. 2002; 40:1845-7.

7. Collins MD, Lawson PA. The genus Abiotrophia (Kawamura et al.) Is not monophyletic: proposal of Granulicatella gen. November, Granulicatella adiacens comb. November, Granulicatella elegans comb. And November Granulicatella balaenopterae comb. November Int J Syst Evol Microbiol, 2000; 50: 365-369.

8. Senn L, Entenza JM, Greub G et al. Bloodstream and endovascular infections due to Abiotrophia defective and Granulicatella species. BMC Infect Dis. 2006; 20:6:9.

9. Ohara-Nemoto Y, Kishi K, Sathe M et al. Infective endocarditis caused by Granulicatella elegans originating in the oral cavity. J Clin Microbiol. 2005; 43:1405-7.

10. Ozenci V, Salaj D, Fang H Westling K, Relapsing endocarditis due to Granulicatella adiacens. Int J Antimicrob Agents, 2009; 33:S47.

11. Perkins A, Osorio S, Serrano $\mathrm{M}$ et al. A case of endocarditis due to Granulicatella adiacens. Clin Microbiol Infect. 2003; 9:576-7.

12. Woo PC, Fung AM, Lau SK et al. Granulicatella adiacens and Abiotrophia defective bacteraemia characterized by $16 \mathrm{~S}$ rRNA gene sequencing. J Med Microbiol. 2003; 52:137-40.

13. Chang SH, Lee CC, Chen SY, Chen IC, Hsieh MR, Chen SC Infectious intracranial aneurysms caused by Granulicatella adiacens. Diagn Microbiol Infect Dis. 2008; 60:201-4.

14. Rosenthal O, Woywodt A, Kirschner P, Haller H. Vertebral osteomyelitis and endocarditis of a pacemaker lead due to Granulicatella (Abiotrophia) adiacens. Infection. 2002; 30:317-9.

15. Eng A, Chen J, Katsivas T. Prosthetic valve endocarditis from Granulicatella adiacens (nutritionally variant streptococci). J Infect. 2005; 51:125-9. 